\title{
The pre-synaptic vesicle protein synaptotagmin is a novel biomarker for Alzheimer's disease
}

Annika Öhrfelt ${ }^{1 *}$ D, Ann Brinkmalm¹,2, Julien Dumurgier ${ }^{3}$, Gunnar Brinkmalm¹, Oskar Hansson ${ }^{4,5}$, Henrik Zetterberg ${ }^{1,2,6}$, Elodie Bouaziz-Amar ${ }^{7}$, Jacques Hugon ${ }^{3}$, Claire Paquet ${ }^{3}$ and Kaj Blennow ${ }^{1,2}$

\begin{abstract}
Background: Synaptic degeneration is a central pathogenic event in Alzheimer's disease that occurs early during the course of disease and correlates with cognitive symptoms. The pre-synaptic vesicle protein synaptotagmin-1 appears to be essential for the maintenance of an intact synaptic transmission and cognitive function. Synaptotagmin-1 in cerebrospinal fluid is a candidate Alzheimer biomarker for synaptic dysfunction that also may correlate with cognitive decline.
\end{abstract}

Methods: In this study, a novel mass spectrometry-based assay for measurement of cerebrospinal fluid synaptotagmin-1 was developed, and was evaluated in two independent sample sets of patients and controls. Sample set I included cerebrospinal fluid samples from patients with dementia due to Alzheimer's disease ( $N=17$, age 52-86 years), patients with mild cognitive impairment due to Alzheimer's disease ( $N=5$, age 62-88 years), and controls ( $N=17$, age 41-82 years). Sample set II included cerebrospinal fluid samples from patients with dementia due to Alzheimer's disease ( $N=24$, age 52-84 years), patients with mild cognitive impairment due to Alzheimer's disease $(N=18$, age 58-83 years), and controls ( $N=36$, age $43-80$ years).

Results: The reproducibility of the novel method showed coefficients of variation of the measured synaptotagmin-1 peptide 215-223 (VPYSELGGK) and peptide 238-245 (HDIIGEFK) of $14 \%$ or below. In both investigated sample sets, the CSF levels of synaptotagmin-1 were significantly increased in patients with dementia due to Alzheimer's disease $(P \leq 0.0001)$ and in patients with mild cognitive impairment due to Alzheimer's disease $(P<0.001)$. In addition, in sample set I the synaptotagmin-1 level was significantly higher in patients with mild cognitive impairment due to Alzheimer's disease compared with patients with dementia due to Alzheimer's disease $(P \leq 0.05)$.

Conclusions: Cerebrospinal fluid synaptotagmin-1 is a promising biomarker to monitor synaptic dysfunction and degeneration in Alzheimer's disease that may be useful for clinical diagnosis, to monitor effect on synaptic integrity by novel drug candidates, and to explore pathophysiology directly in patients with Alzheimer's disease.

Keywords: Alzheimer's disease, Biomarker, Cerebrospinal fluid, Synaptotagmin, Mass spectrometry, Immunopurification, Selected reaction monitoring, Parallel reaction monitoring

\footnotetext{
* Correspondence: annika.ohrfelt@neuro.gu.se

${ }^{1}$ Department of Psychiatry and Neurochemistry, Institute of Neuroscience

and Physiology, The Sahlgrenska Academy at the University of Gothenburg,

Sahlgrenska University Hospital, Mölndal SE-431 80, Sweden

Full list of author information is available at the end of the article
}

\section{Ciomed Central}

(C) 2016 The Author(s). Open Access This article is distributed under the terms of the Creative Commons Attribution 4.0 International License (http://creativecommons.org/licenses/by/4.0/), which permits unrestricted use, distribution, and reproduction in any medium, provided you give appropriate credit to the original author(s) and the source, provide a link to the Creative Commons license, and indicate if changes were made. The Creative Commons Public Domain Dedication waiver (http://creativecommons.org/publicdomain/zero/1.0/) applies to the data made available in this article, unless otherwise stated. 


\section{Background}

Alzheimer's disease is characterized by intracellular neurofibrillary tangles, extracellular accumulation of aggregated amyloid- $\beta$, neuronal degeneration, and synaptic loss [1]. Several cerebrospinal fluid (CSF) biomarkers for Alzheimer's disease are available, including total tau and phosphorylated tau protein, reflecting neurodegeneration and tau pathology, respectively, and amyloid- $\beta_{1-42}$ reflecting deposition of the peptide into plaques $[2,3]$. Numerous studies have consistently shown a marked increase in CSF total tau and phosphorylated tau accompanied by a reduction in amyloid- $\beta_{1-42}$ in Alzheimer's disease, and also in the mild cognitive impairment (MCI) stage of the disease $[2,3]$.

The leading hypothesis on Alzheimer's disease pathogenesis poses that accumulation of amyloid- $\beta$ in the brain is the primary driving force that causes synaptic failure and neurodegeneration, which leads to progressive cognitive deficits $[4,5]$. The accumulation into amyloid- $\beta$ may occur as early as 20-30 years before the first cognitive symptoms [6]. Synaptic degeneration in Alzheimer's disease is related to cognitive decline [7-10] and synaptic loss occurs early in the disease $[11,12]$. Therefore, synaptic biomarkers could be valuable tools for the disease, reflecting synaptic dysfunction, degeneration, or loss. In recent years, promising results have been published for some synaptic biomarkers in CSF, including the pre-synaptic protein synaptosomal-associated protein 25 (SNAP-25) [13] and the post-synaptic protein neurogranin [14] (http://www.alzforum.org/alzbiomarker/meta-analysis/ alzheimers-disease-vs-control-neurogranin-csf). A marked increase of these synaptic CSF markers were found in dementia due to Alzheimer's disease and in MCI due to Alzheimer's disease, with higher CSF levels correlating with more marked future cognitive decline among MCI patients $[13,14]$.

Disruption of synaptic transmission may account for neuronal dysfunction and neurodegeneration. Synaptotagmin-1 is a pre-synaptic calcium sensor protein indispensable for synaptic vesicle exocytosis mediating neurotransmitter release in hippocampal neurons [15-18]. Efficient sustained neurotransmitter release is also dependent on reformation of synaptic vesicles after stimulation by endocytosis, where synaptotagmin- 1 is functioning as an essential vesicle cargo molecule [18]. The crucial function of syntaptotagmin-1 in synaptic transmission [18-20] makes it a potential biomarker candidate reflecting synaptic dysfunction and degeneration in Alzheimer's disease. Indeed, several studies have found a marked reduction of synaptotagmin-1 in typical disease-affected cortical brain regions in Alzheimer's disease [21,22] and a colocalization of synaptotagmin-1 with neuritic plaques [23]. We have previously shown that synaptotagmin is present in CSF
[24], but until now there has not been any available assay for assessment of synaptotagmin in individual CSF samples.

In this study we report a novel mass spectrometrybased assay for measurement of the pre-synaptic protein synaptotagmin-1 in CSF. The aim of the study was to investigate the potential of synaptotagmin-1 as a CSF biomarker in dementia due to Alzheimer's disease and in MCI due to Alzheimer's disease. Because synaptic degeneration is a prominent feature in the brain in Alzheimer's disease, we expected synaptotagmin-1 to be altered in these patients compared with controls and therefore it might serve as a valuable biomarker for Alzheimer's disease.

\section{Methods}

\section{CSF samples}

The reproducibility of the analytical method was investigated in pooled left-over aliquots from decoded CSF samples supplied by the clinical routine section at the Clinical Neurochemistry Laboratory, The Sahlgrenska University Hospital, Mölndal, Sweden, following procedures approved by the Ethical Committee at University of Gothenburg. The quality control CSF pool 1 (QC1 sample) had an amyloid- $\beta_{1-42}$ level of $586 \mathrm{ng} / \mathrm{L}$ and a total tau level below $75 \mathrm{ng} / \mathrm{L}$. The QC2 sample had an amyloid- $\beta_{1-42}$ level of $129 \mathrm{ng} / \mathrm{L}$, a total tau level below $75 \mathrm{ng} / \mathrm{L}$, and a phosphorylated tau level below $15 \mathrm{ng} / \mathrm{L}$.

\section{Selection of the patients}

CSF samples from subjects with either dementia due to Alzheimer's disease or MCI due to Alzheimer's disease or from a neurological control group were obtained from the Research Memory Center at Lariboisière FernandWidal University Hospital APHP. This department is highly experienced in the care management of patients with cognitive disorders and neurodegenerative disease, and has used CSF biomarkers for a long period of time [25-27]. Patients underwent a comprehensive clinical examination including personal medical and family histories, neurological examination, neuropsychological assessment, lumbar puncture with CSF biomarker analysis, and a brain structural imaging study with MRI. A consensus diagnosis was made by several clinicians (neurologists, geriatricians), neuropsychologists, and a biologist who are experts in CSF biomarkers. For each patient, diagnosis was made considering CSF results and according to validated clinical diagnostic criteria for dementia due to Alzheimer's disease [28], MCI due to Alzheimer's disease [29, 30], subjective cognitive impairment [31], and psychiatric disorder (DSM-IV). For all patients, diagnoses were validated in a second step by a neurologist (CP) and a biochemist (EB-A) before selecting CSF samples. In the absence of consensus diagnosis and in 
cases of disagreement about the final diagnosis, patients were not included in the study. According to this method, CSF from subjects with dementia due to Alzheimer's disease, subjects with MCI due to Alzheimer's disease, and neurological controls (no neurodegenerative disorders) was selected. Two sample sets were explored. Recently, the Alzheimer's disease core CSF biomarkers have been included in the research criteria for the diagnosis of both early and manifest $\mathrm{AD}$ by the International Working Group [29] and in the diagnostic guidelines from the National Institute on Aging-Alzheimer's Association [28], respectively. The following cutoff values were used to define a biochemical Alzheimer's disease signature as supportive criteria for dementia due to Alzheimer's disease [28]: amyloid- $\beta_{1-42}(<550 \mathrm{ng} / \mathrm{L})$, total tau $(>400 \mathrm{ng} / \mathrm{L})$, and phosphorylated tau $(>50 \mathrm{ng} / \mathrm{L})$. These criteria was also applied to exclude other diseases and to specify the controls. Sample set I included patients who underwent a lumbar puncture between 2010 and 2012, while sample set II included patients from 2013 to 2015 (Table 1).

\section{CSF collection}

CSF was obtained by lumbar puncture between the L3/ L4 or L4/L5 intervertebral space, using an atraumatic 24-gauge needle, collected in 10-mL polypropylene tubes. Samples were centrifuged at $1800 \times g$ for $10 \mathrm{mi}$ nutes at $+4{ }^{\circ} \mathrm{C}$, were aliquoted in $500 \mu \mathrm{L}$ polypropylene tubes, and were stored at $-80{ }^{\circ} \mathrm{C}$ pending analysis. Samples were frozen at $-80{ }^{\circ} \mathrm{C}$ within $1 \mathrm{~h}$ after collection according to a standardized protocol described in a previous report [26]. A small amount of CSF was used for routine analysis, including total cell count, bacteriologic examination, and total protein and glucose levels.

\section{Analysis of CSF biomarkers}

Amyloid- $\beta_{1-42}$, total tau, and tau phosphorylated at threonine 181 (phosphorylated tau) protein measurements were performed using commercially available assays from Fujirebio (INNOTEST ${ }^{\bullet} \beta$-AMYLOID(1-42), INNOTEST $^{\circ}$ hTAU Ag, and INNOTEST ${ }^{\circ}$ PHOSPHOTAU(181P)) according to the manufacturer's instructions. For the two sample sets, the analysis of these biomarkers was performed in a single hospital laboratory (Lariboisère Hospital Paris) in two runs and averaged results were used for statistical analyses. The quality of CSF evaluations was validated by the Alzheimer's Association quality control program for CSF biomarkers [32].

\section{Antibodies and recombinant protein of synaptotagmin-1}

The monoclonal antibody clone 41.1 recognizing the cytoplasmic portion of synaptotagmin-1 was purchased from Synaptic Systems (Göttingen, Germany). Recombinant protein of synaptotagmin-1 (catalog number TP327252), transcript variant 3, was from OriGene Technologies, Inc. (Rockville, MD, USA).

\section{Immunoprecipitation}

The immunoprecipitation method for CSF samples was performed according to Brinkmalm et al. [13] with minor modifications. Briefly, an aliquot $(1 \mu \mathrm{g})$ of the mouse monoclonal synaptotagmin-1 antibody clone 41.1

Table 1 Demographic data and biomarker CSF levels for the diagnostic groups ${ }^{a}$

\begin{tabular}{|c|c|c|c|}
\hline & Control & MCI-AD & Alzheimer's disease dementia \\
\hline \multicolumn{4}{|l|}{ Sample set I } \\
\hline Number (men/women) & $17(7 / 10)$ & $5(1 / 4)$ & $17(5 / 12)$ \\
\hline Age (years) & $60(53-67), P=0.02^{c}$ & $78(73-81), P=0.02^{\mathrm{b}}$ & $65(58-81)$ \\
\hline MMSE & $27(24-28)$ & $27(27-28)$ & $21(16-23), P=0.00001^{b}, P=0.004^{c}$ \\
\hline Amyloid- $\beta_{1-42}$ (ng/L) & $838(697-998), P=0.001^{c}$ & $539(316-582), P=0.001^{b}$ & $398(319-483), P<0.00001^{b}$ \\
\hline Total tau (ng/L) & $180(126-206), P=0.00008^{c}$ & $1000(766-1078), P=0.00008^{b}$ & $602(442-769), P<0.00001^{b}$ \\
\hline Phosphorylated tau (ng/L) & $39(35-42), P=0.00008^{c}$ & $143(97-180), P=0.00008^{b}$ & $89(75-119), P<0.00001^{b}$ \\
\hline \multicolumn{4}{|l|}{ Sample set II } \\
\hline Number (men/women) & $36(13 / 23)$ & $18(5 / 13)$ & $24(7 / 17)$ \\
\hline Age (years) & $62(55-69), P=0.001^{c}$ & $70(69-78), P=0.001^{\mathrm{b}}$ & $68(64-72), P=0.02^{\mathrm{b}}$ \\
\hline MMSE & $28(26-29)$ & $27(26-28)$ & $22(17-24), P=0.00001^{b}, P=0.00001^{c}$ \\
\hline Amyloid- $\beta_{1-42}(\mathrm{ng} / \mathrm{L})$ & $971(844-1110), P<0.00001^{c}$ & $558(377-667), P<0.00001^{b}$ & $497(459-571), P<0.00001^{b}$ \\
\hline Total tau (ng/L) & $197(157-227), P<0.00001^{c}$ & $570(520-717), P<0.00001^{\mathrm{b}}$ & $623(527-799), P<0.00001^{b}$ \\
\hline Phosphorylated tau (ng/L) & $40(32-46), P<0.00001^{c}$ & $86(79-104), P<0.00001^{b}$ & $91(73-115), P<0.00001^{b}$ \\
\hline
\end{tabular}

${ }^{a}$ Data given as median (interquartile range) unless otherwise indicated. Statistical differences were determined using nonparametric tests

${ }^{\mathrm{b}}$ Compared with controls

${ }^{c}$ Compared with $\mathrm{MCl}-\mathrm{AD}$

$M C I-A D$ mild cognitive impairment due to Alzheimer's disease, MMSE Mini-Mental State Examination 
$(1 \mathrm{~g} / \mathrm{L})$ or IgG from murine serum $(1 \mathrm{~g} / \mathrm{L}$, a negative control; Sigma-Aldrich) was separately added to $50 \mu \mathrm{L}$ magnetic Dynabeads M-280 Sheep anti-mouse IgG (Invitrogen Corporation) and incubated for $1 \mathrm{~h}$ on a rocking platform at room temperature. The beads were washed three times with $1 \mathrm{ml}$ of phosphate-buffered saline (PBS). The antibodies were cross-linked using $20 \mathrm{mM}$ dimethyl pimelimidate dihydrochloride (Sigma-Aldrich) and 0.2 M triethanolamine ( $\mathrm{pH}$ 8.2; Sigma-Aldrich) according to the manufacturer's product description. The cross-linked beads were washed twice in PBS and were blocked with Roti-Block (Carl Roth) for $1 \mathrm{~h}$ on a rocking platform at room temperature. CSF samples $(250 \mu \mathrm{L})$ were adjusted with $5 \%$ Tween 20 and PBS to a final concentration of $0.05 \%$ Tween 20 and a final volume of $1 \mathrm{~mL}$. Samples and magnetic beads were incubated overnight on a rocking platform at $+4{ }^{\circ} \mathrm{C}$. The magnetic beads/sample solution was transferred to the KingFisher magnetic particle processor (Thermo Fisher Scientific), tube 1 . The following three wash steps (tubes 2-4) were conducted for $10 \mathrm{~s}$ in $1 \mathrm{~mL}$ of each washing buffer: (tube 2) $0.025 \%$ Tween 20 in PBS, (tube 3) PBS, and (tube 4) $50 \mathrm{mM}$ ammonium hydrogen carbonate $\left(\mathrm{NH}_{4} \mathrm{HCO}_{3}\right.$, $\mathrm{pH}$ 8.0). Synaptotagmin-1 was then eluted from the beads by adding $100 \mu \mathrm{L}$ of $0.5 \%$ formic acid (tube 5) for $4 \mathrm{~min}$. The eluted fractions were transferred to $0.65 \mathrm{~mL}$ prelubricated Costar Microcentrifuge Tube (Fisher Scientific) and dried in a vacuum centrifuge.

\section{Protein digestion and addition of heavy-isotope-labeled peptide standards}

Two isotopically labeled custom-made peptides containing U-13C6, U-15 N2-lysine[K] (aa 215-223, VPYSELGG[K] and aa 238-245, HDIIGEFK HeavyPeptide FasTrack 1 standards; Thermo Fisher Scientific) were dissolved in MilliQ water $(\sim 500 \mathrm{pmol} / \mu \mathrm{L})$, mixed, and diluted in $50 \mathrm{mM} \mathrm{NH} \mathrm{HCO}_{3}$ to a final concentration of $\sim 10 \mathrm{fmol} / \mu \mathrm{L}$. The dried immunoprecipitated CSF samples were dissolved in $25 \mu \mathrm{L}$ of a mixture of trypsin and labeled peptides $(1 \mu \mathrm{g}$ Sequencing Grade Modified Trypsin (Promega) dissolved in $0.01 \%$ aqueous $\mathrm{HCl}(0.1 \mathrm{~g} / \mathrm{L})$ and diluted to $5 \mathrm{mg} / \mathrm{L}$ in isotopically labeled peptide mixture in $50 \mathrm{mM} \mathrm{NH} \mathrm{HCO}_{3}, \mathrm{pH} \approx 7.8$ (see earlier)), vortexed carefully, and incubated at $+37{ }^{\circ} \mathrm{C}$ overnight. To stop the enzymatic activity, $5 \mu \mathrm{L}$ of $10 \%$ aqueous FA was added. The samples were centrifuged $(16,900 \times g$, $10 \mathrm{~min},+4^{\circ} \mathrm{C}$ ) and $27 \mu \mathrm{L}$ of each sample was transferred to LC-vials (SUN-SRi).

\section{High-resolution parallel reaction monitoring analyses}

High-resolution parallel reaction monitoring (HR-PRM) analyses were performed on a Q Exactive quadrupoleorbitrap mass spectrometer (Thermo Fisher Scientific) coupled to an Ultimate 3000 standard liquid chromatography system (Thermo Fisher Scientific). The samples $(25 \mu \mathrm{L})$ were loaded directly onto a Hypersil GOLD HPLC C18 column (length $100 \mathrm{~mm}$; inner diameter $2.1 \mathrm{~mm}$; particle size $1.9 \mu \mathrm{m}$; Thermo Fisher Scientific) with $0.1 \%$ aqueous formic acid at $100 \mu \mathrm{L} / \mathrm{min}$. Mobile phases were: A, $0.1 \%$ formic acid in water $(\mathrm{v} / \mathrm{v})$; and $\mathrm{B}$, $0.1 \%$ formic acid and $84 \%$ acetonitrile in water $(\mathrm{v} / \mathrm{v})$. The peptides were eluted off the column using the following linear gradient steps: $1 \mathrm{~min}, 0 \% \mathrm{~B} ; 3 \mathrm{~min}, 12 \%$ B; $11 \mathrm{~min}, 40 \% \mathrm{~B}$; and $13 \mathrm{~min}, 100 \% \mathrm{~B}$. The IonMax electrospray ion source settings were: spray voltage, $+4100 \mathrm{~V}$; capillary temperature, $+320{ }^{\circ} \mathrm{C}$; sheath gas pressure setting, 25 arbitrary units; auxiliary gas pressure setting, 10 arbitrary units; and heater temperature, $+300^{\circ} \mathrm{C}$. The instrument was set to acquire scheduled pairs of SIM scans and subsequently all ion fragmentation scans (isolation window $8.0 \mathrm{~m} / z$ ) in profile mode, allowing simultaneous detection of both the synaptotagmin peptide and the corresponding isotopically labeled peptide standard. The settings were common for both scan types and were as follows: resolution setting, 35,000; AGC target, 1e6; maximum injection time, 120 ms. Data acquisition and analysis were performed with Xcalibar software version 2.2 SP1.48 (Thermo Fisher Scientific).

\section{Assay reproducibility}

The intra-day variation of synaptotagmin was determined using two QC samples (QC1 and QC2). The immunoprecipitation of synaptotagmin-1 from the QC samples were performed on different days but analyzed on the same occasion. Assessment of reproducibility was performed on two different occasions, and QC samples were randomized in between samples from sample set I (first occasion) and sample set II (second occasion).

\section{Statistical analysis}

Because most of the analytes were not normally distributed (Shapiro-Wilk test, $P<0.05$ ), nonparametric statistics were used for analysis. Data are given as the median (interquartile range). Differences between more than two groups were assessed with Kruskal-Wallis test. Statistically significant results $(P<0.05)$ were followed by Mann-Whitney $U$ tests to investigate group differences. Receiver operating characteristic (ROC) curves were performed on each subject group on the tryptic peptides of synaptotagmin-1 in order to assess their diagnostic value. For each tryptic peptide of synaptotagmin, the area under the curve and a $95 \%$ confidence interval was calculated using GraphPad Prism 5. The correlation coefficients (rho) were calculated using the Spearman twotailed correlation test. SPSS 20.0 was employed for most of the statistical analyses. 


\section{Results}

\section{Assay performance}

The reproducibility of the novel method showed coefficients of variation (CV) of the measured synaptotagmin-1 peptide 215-223 (VPYSELGGK) and peptide 238-245 (HDIIGEFK) of $14 \%$ or below (Additional file 1: Table S1). The investigated tryptic peptides correlated with each other in the groups of controls (rho $=0.971, P<0.00001$, sample set I; and rho $=0.995, P<0.00001$, sample set II), MCI due to Alzheimer's disease (rho $=0.988, P<0.00001$, sample set II; sample set I was too small for statistical evaluation), and dementia due to Alzheimer's disease (rho $=0.980, P<0.00001$, sample set $\mathrm{I}$; and rho $=0.995$, $P<0.00001$, sample set II) (Table 2).

\section{Clinical studies}

\section{Demographics}

Table 1 presents the demographic characteristics of the groups. Sample set I consisted of five patients with MCI due to Alzheimer's disease (one man and four women, age 62-88 years), 17 patients with dementia due to Alzheimer's disease (five men and 12 women, age 52-86 years), and 17 neurological controls (seven men and 10 women, age 41-82 years). The replication sample set (sample set II) consisted of 18 patients with MCI due to Alzheimer's disease (five men and 13 women, age 58-83 years), 24 patients with dementia due to Alzheimer's disease (seven men and 17 females, age 52-84 years), and 36 neurological controls (13 men and 23 women, age 43-80 years). In sample set I, the patients with MCI due to Alzheimer's disease were older than the subjects with controls. Both patients with MCI due to Alzheimer's disease and patients with dementia due to Alzheimer's disease were significantly older than the subjects with controls in sample set II. All patients from the neurological control group had cognitive complaints (group I, $N=14$ and group II, $N=25$ ) or had psychiatric diagnoses (group I, $N=3$ and group II, $N=11$ ). To rule out preclinical Alzheimer's disease in the neurological control group, only cases with normal CSF levels amyloid- $\beta_{1-42}$ $(>550 \mathrm{ng} / \mathrm{L})$, total tau $(<400 \mathrm{ng} / \mathrm{L})$, and phosphorylated tau $(<50 \mathrm{ng} / \mathrm{L})$ were included.

The CSF levels of the investigated tryptic peptides of synaptotagmin-1 were significantly higher in patients with MCI due to Alzheimer's disease and patients with dementia due to Alzheimer's disease compared with controls (Fig. 1). In sample set I, the tryptic peptides of synaptotagmin-1 were significantly higher in patients with MCI due to Alzheimer's disease compared with patients with dementia due to Alzheimer's disease (Fig. 1a, b).

Each of the tryptic peptide assays of synaptotagmin-1 (215-223 and 238-245) could differentiate MCI due to Alzheimer's disease from controls in both sample sets, with area under the curve of $0.988(0.952-1.025)(P=0.001)$ and
1.000 (1.000-1.000) $(P=0.0009)$ (sample set I), respectively, and of $0.813(0.692-0.934)(\mathrm{P}=0.0002)$ and $0.801(0.676-$ 0.926) $(P=0.0003)$ (sample set II), respectively (Fig. 2a). The tryptic peptide assays of synaptotagmin-1 (215-223 and 238-245) could also each differentiate dementia due to Alzheimer's disease from controls in both sample sets, with area under the curve of $0.886(0.755-1.017)(P=0.0001)$ and $0.893(0.770-1.016)(P<0.0001)$ (sample set I), respectively, and of $0.815(0.703-0.926)(P<0.0001)$ and 0.795 $(0.679-0.912) \quad(P=0.0001) \quad$ (sample set $\mathrm{II})$, respectively (Fig. 2b).

No statistically significant correlations between age and the levels of the tryptic peptides of synaptotagmin were observed in any of the investigated groups (Table 2). There were no statistical significant correlations between CSF synaptotagmin-1 and Mini-Mental State Examination (MMSE) scores in any group.

The CSF levels of synaptotagmin (tryptic peptides 215-223 and 238-245) correlated with the levels of total tau and phosphorylated tau in both the control group (sample sets I and II) and in patients with dementia due to Alzheimer's disease (sample sets I and II), but not in patients with MCI due to Alzheimer's disease (sample set II) (Table 2). Synaptotagmin-1 (215-223) correlated with amyloid- $\beta_{1-42}$ in the control group (rho $=0.515$, $P=0.04$ and rho $=0.381, P=0.02$, respectively), but not in patients with either MCI due to Alzheimer's disease or dementia due to Alzheimer's disease (Table 2). Synaptotagmin-1 (238-245) correlated only with amyloid$\beta_{1-42}($ rho $=0.348, P=0.04$ ) in the control group of sample set II, while there were no correlations within other investigated groups (Table 2).

\section{Discussion}

In this study, we investigated the potential of synaptotagmin-1 as a CSF biomarker in dementia due to Alzheimer's disease and in MCI due to Alzheimer's disease. Therefore, we developed novel assays for the measurement of synaptotagmin-1, which is a pre-synaptic vesicle protein indispensable for the regulation of synaptic transmission and for sustaining intact cognitive function. In the present study, we report that the levels of synaptotagmin-1 were significantly increased in patients with MCI due to Alzheimer's disease and patients with dementia due to Alzheimer's disease compared with controls, supporting that synaptotagmin-1 could be an early marker for Alzheimer's disease.

The novel assay combines immunoprecipitation, tryptic digestion and finally mass spectrometry (HR-PRM) for analyses of the CSF levels of synaptotagmin, which is a similar approach to that we applied successfully for quantification of SNAP-25 in CSF [13]. Synaptotagmin-1 consists of an $\mathrm{N}$-terminal intravesicular domain, a transmembrane part, and a large cytoplasmic sequence 
Table 2 Correlation between age, MMSE, and biomarker levels for the diagnostic groups ${ }^{2}$

\begin{tabular}{|c|c|c|}
\hline & Synaptotagmin, 215-223 & Synaptotagmin, 238-245 \\
\hline \multicolumn{3}{|l|}{ Sample set I, control $(N=17)$} \\
\hline Age & N.S. & N.S. \\
\hline MMSE & N.S. & N.S. \\
\hline Amyloid- $\beta_{1-42}$ (ng/L) & rho $=0.515, P=0.04$ & N.S. \\
\hline Total tau (ng/L) & rho $=0.739, P=0.001$ & rho $=0.749, P=0.001$ \\
\hline Phosphorylated tau (ng/L) & rho $=0.770, P=0003$ & rho $=0.708, P=0.001$ \\
\hline Synaptotagmin, 215-223 & - & rho $=0.971, P<0.00001$ \\
\hline Synaptotagmin, 238-245 & rho $=0.971, P<0.00001$ & - \\
\hline \multicolumn{3}{|c|}{ Alzheimer's disease dementia $(N=17)$} \\
\hline Age & N.S. & N.S. \\
\hline MMSE & N.S. & N.S. \\
\hline Amyloid- $\beta_{1-42}$ (ng/L) & N.S. & N.S. \\
\hline Total tau (ng/L) & rho $=0.540, P=0.03$ & rho $=0.610, P=0.009$ \\
\hline Phosphorylated tau (ng/L) & rho $=0.586, P=0.01$ & rho $=0.656, P=0.004$ \\
\hline Synaptotagmin, 215-223 & - & rho $=0.980, P<0.00001$ \\
\hline Synaptotagmin, 238-245 & rho $=0.980, P<0.00001$ & - \\
\hline \multicolumn{3}{|l|}{ Sample set II, control $(N=36)$} \\
\hline Age & N.S. & N.S. \\
\hline MMSE & N.S. & N.S. \\
\hline Amyloid- $\beta_{1-42}$ (ng/L) & rho $=0.381, P=0.02$ & rho $=0.348, P=0.04$ \\
\hline Total tau (ng/L) & rho $=0.641, P=0.00003$ & rho $=0.633, P=0.00004$ \\
\hline Phosphorylated tau (ng/L) & rho $=0.687, P<0.00001$ & rho $=0.683, P<0.00001$ \\
\hline Synaptotagmin, 215-223 & - & rho $=0.995, P<0.00001$ \\
\hline Synaptotagmin, 238-245 & rho $=0.995, P<0.00001$ & - \\
\hline \multicolumn{3}{|l|}{$\mathrm{MCl}-\mathrm{AD}(\mathrm{N}=18)$} \\
\hline Age & N.S. & N.S. \\
\hline MMSE & N.S. & N.S. \\
\hline Amyloid- $\beta_{1-42}$ (ng/L) & N.S. & N.S. \\
\hline Total tau (ng/L) & N.S. & N.S. \\
\hline Phosphorylated tau (ng/L) & N.S. & N.S. \\
\hline Synaptotagmin, 215-223 & - & rho $=0.988, P<0.00001$ \\
\hline Synaptotagmin, 238-245 & rho $=0.988, P<0.00001$ & - \\
\hline \multicolumn{3}{|c|}{ Alzheimer's disease dementia $(N=24)$} \\
\hline Age & N.S. & N.S. \\
\hline MMSE & N.S. & N.S. \\
\hline Amyloid- $\beta_{1-42}$ (ng/L) & N.S. & N.S. \\
\hline Total tau (ng/L) & rho $=0.655, P=0.001$ & rho $=0.675, P=0.0003$ \\
\hline Phosphorylated tau (ng/L) & rho $=0.653, P=001$ & rho $=0.673, P=0.0003$ \\
\hline Synaptotagmin, 215-223 & - & rho $=0.995, P<0.00001$ \\
\hline Synaptotagmin, 238-245 & rho $=0.995, P<0.00001$ & - \\
\hline
\end{tabular}

${ }^{a}$ Correlations presented by the Spearman's rank correlation coefficient (rho). Nonsignificant (N.S., $P>0.05$ ) correlations were not reported $M C I-A D$ mild cognitive impairment due to Alzheimer's disease, MMSE Mini-Mental State Examination 

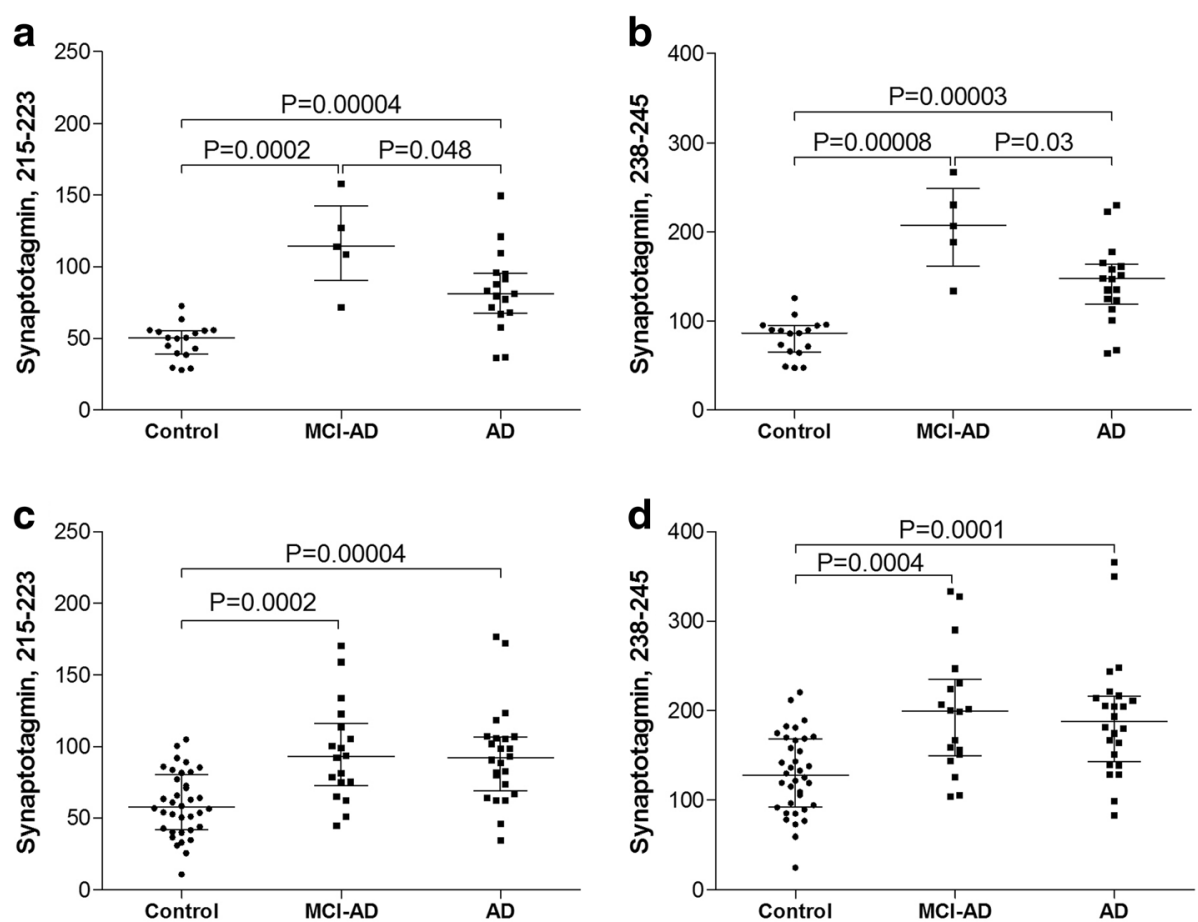

Fig. 1 Targeted HR-PRM-MS analyses of synaptotagmin in human CSF. Individual values for the HR-PRM-MS measured peak area ratios ((endogenous peptide/labeled peptide standard) $\times 1000$ ) of immunoprecipitated synaptotagmin in CSF samples within sample set I (a, b) and sample set II (c, d). The four panels depict the measured levels of two tryptic peptides of synaptotagmin, 215-223 (VPYSELGGK) (a, c) and 238-245 (HDIIGEFK) (b, d). $A D$ dementia due to Alzheimer's disease, MCl-AD mild cognitive impairment due to Alzheimer's disease

that contains two calcium (C2) binding domains. Both tryptic peptides of the novel assays are located in the first (C2A) of two calcium-binding domains of synaptotagmin-1, domains of the protein that are essential for appropriate synaptic transmission [16-18]. Furthermore, the novel mass spectrometry-based method allows selective measurements of the synaptotagmin- 1 isoform. Since the 17 members of the synaptotagmin family are differently expressed throughout the body $[33,34]$ and these isoforms exhibit diverse biological functions, the ability to discriminate between them is certainly of great importance for a valuable synaptic biomarker.

We found that the CSF levels of synaptotagmin-1 were consistently elevated in patients with dementia due to Alzheimer's disease compared with controls in two separate sample sets. Additionally, synaptotagmin-1 was increased already in MCI due to Alzheimer's disease, supporting the notion that this synaptic marker might be an early marker for Alzheimer's disease. These findings are in accordance with our previous findings of the pre-synaptic marker SNAP-25 [13] and the post-synaptic marker neurogranin [14]. Furthermore, synaptotagmin-1 was even higher in MCI due to Alzheimer's disease compared with dementia due to Alzheimer's disease, suggesting that synaptic dysfunction and degeneration can be identified before the onset of clinical dementia [11, 12]. However, because this latter finding was only supported by data from sample set I, this notion needs to be taken with caution and needs to be reproduced in a larger set of patients.

Each measured tryptic peptide of synaptotagmin-1 (215-223 and 238-245) could differentiate dementia due to Alzheimer's disease from controls and MCI due to Alzheimer's disease from controls to a similar magnitude, in the respective sample sets. The tryptic peptides of synaptotagmin-1 assays also correlated with each other in all investigated groups (controls, MCI due to Alzheimer's disease, and dementia due to Alzheimer's disease) in the respective sample sets. Moreover, the reproducibility of the novel method $(\mathrm{CV})$ was less than $14 \%$. Altogether these findings support the notion that each tryptic peptide corresponds to the same synaptotagmin-1 molecule.

In both sample sets, the MCI due to Alzheimer's disease patients were significantly older than the controls. In sample set I the patients with dementia due to Alzheimer's disease and the controls were age matched, while the patients with dementia due to Alzheimer's disease were significantly older than the controls in sample set II. On the other hand, there were no statistically significant correlations between age and the levels of synaptotagmin-1 in 

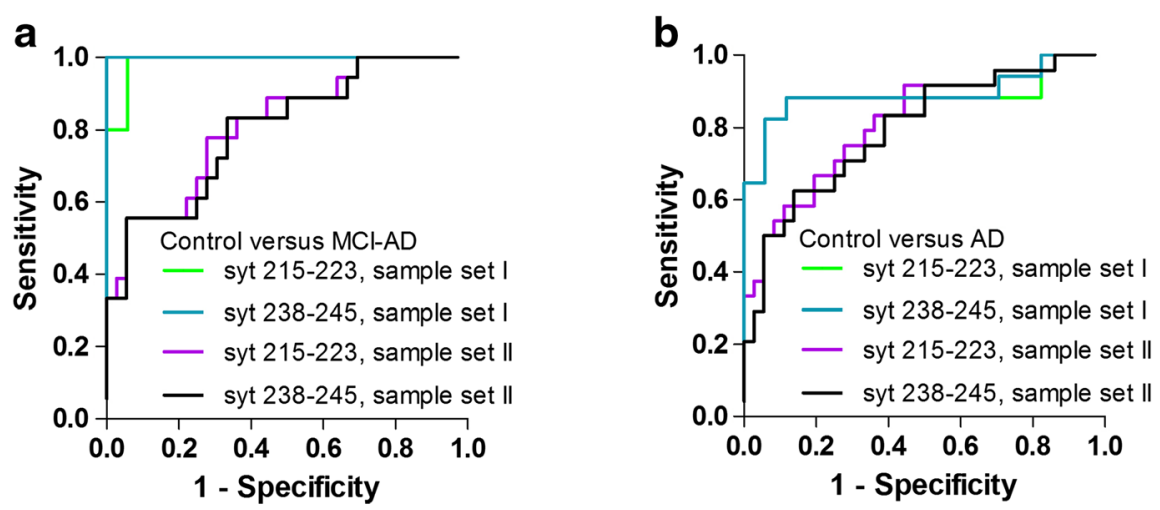

C

\begin{tabular}{|lll|}
\hline & Control versus MCI-AD & Control versus AD \\
\hline Sample se I & $\mathrm{N}=17$ and $\mathrm{N}=5$ & $\mathrm{~N}=17$ and $\mathrm{N}=17$ \\
Synaptotagmin, 215-223 & $0.988(0.952-1.025), \mathrm{P}=0.001$ & $0.886(0.755-1.017), \mathrm{P}=0.0001$ \\
Synaptotagmin, 238-245 & $1.000(1.000-1.000), \mathrm{P}=0.0009$ & $0.893(0.770-1.016), \mathrm{P}<0.0001$ \\
\hline Sample set II & $\mathrm{N}=36$ and N=18 & $\mathrm{N}=36$ and $\mathrm{N}=24$ \\
Synaptotagmin, 215-223 & $0.813(0.692-0.934), \mathrm{P}=0.0002$ & $0.815(0.703-0.926), \mathrm{P}<0.0001$ \\
Synaptotagmin, 238-245 & $0.801(0.676-0.926), \mathrm{P}=0.0003$ & $0.795(0.679-0.912), \mathrm{P}=0.0001$ \\
\hline
\end{tabular}

Fig. 2 ROC curve analysis of synaptotagmin in human CSF. ROC curve analysis for synaptotagmin 215-223 (green and pink) and synaptotagmin 238-245 (turquoise and black) in CSF for differentiation of MCI due to Alzheimer's disease (MCI-AD) from controls in sample set I and sample set II, respectively (a). ROC curve analysis for synaptotagmin 215-223 (green and pink) and synaptotagmin 238-245 (turquoise and black) in CSF for differentiation of dementia due to Alzheimer's disease (AD) from controls in sample set I and sample set II, respectively (b). The area under the curve (95\% confidence interval) is shown in the included table (c). AD dementia due to Alzheimer's disease, MCl-AD mild cognitive impairment due to Alzheimer's disease (Color figure online)

any of the investigated groups, suggesting that the CSF synaptotagmin-1 levels not are affected by age within the age range of this study.

The mechanism for release of synaptotagmin-1 into CSF is not known. However, it is interesting to note that previous studies showed that $\mathrm{C} 2$ domains may penetrate a cell membrane upon calcium binding $[35,36]$, which could be a possible mechanism of liberation into CSF. Herein, we found that synaptotagmin-1 correlated with the levels of total tau and phosphorylated tau both in the control group and in patients with dementia due to Alzheimer's disease in both investigated sample sets. CSF total tau has previously been suggested to be a general marker of damage to cortical nonmyelinated neurons [2]. In contrast, phosphorylated tau might be a more specific marker for Alzheimer's disease [2], since high CSF levels of phosphorylated tau have been found to correlate with the accumulation of cortical neurofibrillary tangles $[37,38]$. To summarize, our results suggest that synaptotagmin-1 might be a specific marker for dementia due to Alzheimer's disease that to some extent also might reflect general neurodegeneration.

The fact that synaptic loss is the main pathological feature of dementia due to Alzheimer's disease that correlates with cognitive decline, together with the notion that synaptotagmin-1 is directly involved in the regulation of neurotransmitter release $[16,18]$, make synaptotagmin-1 a potential CSF biomarker to follow progression of clinical symptoms. In the present study, there were no correlations between cognitive decline and the tryptic peptides of synaptotagmin-1 in any of the examined groups, possibly due to the small size of the clinical sample sets. Further studies are therefore needed to investigate whether synaptotagmin-1 in CSF could be used for assessment of the future rate of cognitive decline.

The strength of the present study is that we present robust assays for assessment of the CSF levels of synaptotagmin-1 in two independent sample sets. To our knowledge this is the first study investigating the potential of synaptotagmin-1 as a CSF biomarker for Alzheimer's disease. One limitation of the present study is the crosssectional design of the clinical study that complicates the investigation of possible association between CSF synaptotagmin and synaptic degeneration. This question has to be addressed by longitudinal measurements of synaptotagmin to investigate whether the levels are changed over time and whether it correlates with cognitive alterations in patients with Alzheimer's disease. Another limitation could probably be that all clinical samples were analyzed as single samples. Since the analytical reproducibility of the method $(\mathrm{CV}<14 \%)$ yields very similar results for $\mathrm{du}$ plicates while the measured levels vary considerably between patients, we decided to include as many different patients as possible and thereby try to obtain a more representative picture of the biological variation rather than the analytical. 


\section{Conclusions}

We present a novel method for measurement of the pre-synaptic protein synaptotagmin-1 in CSF samples. Synaptotamin-1 concentrations were increased in patients with MCI due to Alzheimer's disease and patients with dementia due to Alzheimer's disease compared with controls, supporting the notion that synaptotagmin-1 could be a valuable differential biomarker both in early Alzheimer's disease and after manifestation of the disease.

\section{Additional file}

Additional file 1: Table S1. Presenting the intra-assay varibility for the QC samples. (DOCX $46 \mathrm{~kb}$ )

\section{Abbreviations}

CV: Coefficients of variation; MCl: Mild cognitive impairment; MMSE: Mini-Mental State Examination; PBS: Phosphate-buffered saline; SNAP-25: Synaptosomalassociated protein 25; ROC: Receiver operating characteristic

\section{Acknowledgements}

The authors are grateful to Rita Persson, Prof. Jean-Louis Laplanche, Dr Sarah Gourmaud, and Sylvie Thomasseau for their technical assistance.

\section{Funding}

This work was supported by grants from the Swedish Brain Power Consortium, Swedish Alzheimer Foundation, the Swedish Brain Foundation, Swedish Research Council, European Research Council, ALF, the Knut and Alice Wallenberg Foundation, Demensfonden, Eivind och Elsa K:son Sylvans stiftelse, the Wolfson Foundation, Märtha och Gustaf Ågrens stiftelse, Stohnes stiftelse, Stiftelsen Gamla Tjänarinnor, Magn. Bergvalls stiftelse, Svenska Läkaresällskapet, the Torsten Söderberg Foundation at the Royal Swedish Academy of Sciences, Åhlén-stiftelsen, and BMBF BIOMARK-APD (DLR 01ED1203 J).

\section{Authors' contributions}

$A O ̈$ and $K B$ performed the study design, interpretation of the results, and writing of the manuscript draft. $A B, J D, G B, O H, H Z, E B-A, J H$, and $C P$ contributed to the study concept and design, and to critical revision of the manuscript for important intellectual content. $A B$ and $A O ̈$ performed the experiments and analyzed and compiled data. All authors read and approved the final manuscript.

\section{Competing interests}

The authors declare that they have no competing interests.

\section{Ethics approval and consent to participate}

The study was approved by the Ethics Committee of Paris Diderot University Hospital (Bichat Hospital). All patients or caregivers gave their written informed consents for research, which was conducted in accordance with the Helsinki Declaration.

\footnotetext{
Author details

'Department of Psychiatry and Neurochemistry, Institute of Neuroscience and Physiology, The Sahlgrenska Academy at the University of Gothenburg, Sahlgrenska University Hospital, Mölndal SE-431 80, Sweden. ${ }^{2}$ Clinical Neurochemistry Laboratory, Sahlgrenska University Hospital, Mölndal, Sweden. ${ }^{3}$ Centre Mémoire de Ressources et de Recherche (CMRR) Paris Nord lle de France, INSERM UMR-S942, Groupe Hospitalier Lariboisière Fernand-Widal Saint-Louis, Paris, France. ${ }^{4}$ Clinical Memory Research Unit, Department of Clinical Sciences, Lund University, Malmö, Sweden. ${ }^{5}$ Memory Clinic, Skåne University Hospital, Malmö, Sweden. ${ }^{6}$ Department of Molecular Neuroscience, UCL Institute of Neurology, London, UK. ${ }^{7}$ Service de Biochimie Groupe Hospitalier Lariboisiere FW Saint-Louis, APHP, Université Paris Diderot, 75010 Paris, France.
}

Received: 7 July 2016 Accepted: 5 September 2016

Published online: 03 October 2016

\section{References}

1. Blennow K, de Leon MJ, Zetterberg H. Alzheimer's disease. Lancet. 2006; 368(9533):387-403. doi:10.1016/S0140-6736(06)69113-7.

2. Blennow K, Hampel H, Weiner M, Zetterberg $H$. Cerebrospinal fluid and plasma biomarkers in Alzheimer disease. Nat Rev Neurol. 2010;6(3):131-44. doi:10.1038/nrneurol.2010.4.

3. Olsson B, Lautner R, Andreasson U, Ohrfelt A, Portelius E, Bjerke M, et al. CSF and blood biomarkers for the diagnosis of Alzheimer's disease: a systematic review and meta-analysis. Lancet Neurol. 2016;15(7):673-84. doi:10.1016/ S1474-4422(16)00070-3.

4. Hardy J, Selkoe DJ. The amyloid hypothesis of Alzheimer's disease: progress and problems on the road to therapeutics. Science. 2002;297(5580):353-6. doi:10.1126/science.1072994.

5. Selkoe DJ. Alzheimer's disease is a synaptic failure. Science. 2002;298(5594): 789-91. doi:10.1126/science.1074069.

6. Jansen WJ, Ossenkoppele R, Knol DL, Tijms BM, Scheltens P, Verhey FR, et al. Prevalence of cerebral amyloid pathology in persons without dementia: a meta-analysis. JAMA. 2015;313(19):1924-38. doi:10.1001/jama.2015.4668.

7. Blennow K, Bogdanovic N, Alafuzoff I, Ekman R, Davidsson P. Synaptic pathology in Alzheimer's disease: relation to severity of dementia, but not to senile plaques, neurofibrillary tangles, or the ApoE4 allele. J Neural Transm (Vienna). 1996;103(5):603-18.

8. DeKosky ST, Scheff SW. Synapse loss in frontal cortex biopsies in Alzheimer's disease: correlation with cognitive severity. Annals Neurol. 1990;27(5):457-64. do::10.1002/ana.410270502

9. Terry RD, Masliah E, Salmon DP, Butters N, DeTeresa R, Hill R, et al. Physical basis of cognitive alterations in Alzheimer's disease: synapse loss is the major correlate of cognitive impairment. Annals Neurol. 1991;30(4):572-80. doi:10.1002/ana.410300410

10. Scheff SW, Price DA. Synaptic pathology in Alzheimer's disease: a review of ultrastructural studies. Neurobiol Aging. 2003;24(8):1029-46

11. Davies CA, Mann DM, Sumpter PQ, Yates PO. A quantitative morphometric analysis of the neuronal and synaptic content of the frontal and temporal cortex in patients with Alzheimer's disease. J Neurol Sci. 1987:78(2):151-64.

12. Masliah E, Mallory M, Alford M, DeTeresa R, Hansen LA, McKeel Jr DW, et al. Altered expression of synaptic proteins occurs early during progression of Alzheimer's disease. Neurology. 2001;56(1):127-9.

13. Brinkmalm A, Brinkmalm G, Honer WG, Frolich L, Hausner L, Minthon L, et al. SNAP-25 is a promising novel cerebrospinal fluid biomarker for synapse degeneration in Alzheimer's disease. Mol Neurodegener. 2014;9:53. doi:10.1186/1750-1326-9-53.

14. Kvartsberg H, Duits FH, Ingelsson M, Andreasen N, Ohrfelt A, Andersson K, et al. Cerebrospinal fluid levels of the synaptic protein neurogranin correlates with cognitive decline in prodromal Alzheimer's disease. Alzheimers Dement. 2015:11(10):1180-90. doi:10.1016/j.jalz.2014.10.009.

15. Jahn R, Fasshauer D. Molecular machines governing exocytosis of synaptic vesicles. Nature. 2012:490(7419):201-7. doi:10.1038/nature11320.

16. Sudhof TC, Rizo J. Synaptotagmins: C2-domain proteins that regulate membrane traffic. Neuron. 1996:17(3):379-88.

17. Lynch KL, Gerona RR, Larsen EC, Marcia RF, Mitchell JC, Martin TF. Synaptotagmin C2A loop 2 mediates Ca2+-dependent SNARE interactions essential for Ca2+-triggered vesicle exocytosis. Mol Biol Cell. 2007;18(12): 4957-68. doi:10.1091/mbc.E07-04-0368.

18. Geppert M, Goda Y, Hammer RE, Li C, Rosahl TW, Stevens CF, et al. Synaptotagmin I: a major Ca2+ sensor for transmitter release at a central synapse. Cell. 1994;79(4):717-27.

19. Bacaj T, Wu D, Burre J, Malenka RC, Liu X, Sudhof TC. Synaptotagmin-1 and -7 are redundantly essential for maintaining the capacity of the readilyreleasable pool of synaptic vesicles. PLoS Biol. 2015;13(10):e1002267. doi:10. 1371/journal.pbio.1002267.

20. Baker K, Gordon SL, Grozeva D, van Kogelenberg M, Roberts NY, Pike M, et al. Identification of a human synaptotagmin-1 mutation that perturbs synaptic vesicle cycling. J Clin Invest. 2015;125(4):1670-8. doi:10.1172/JCI79765.

21. Davidsson P, Blennow K. Neurochemical dissection of synaptic pathology in Alzheimer's disease. Int Psychogeriatr. 1998;10(1):11-23.

22. Yoo BC, Cairns N, Fountoulakis M, Lubec G. Synaptosomal proteins, betasoluble N-ethylmaleimide-sensitive factor attachment protein (beta-SNAP), 
gamma-SNAP and synaptotagmin I in brain of patients with Down syndrome and Alzheimer's disease. Dement Geriatr Cogn Disord. 2001;12(3):219-25.

23. Masliah E, Honer WG, Mallory M, Voigt M, Kushner P, Hansen L, et al. Topographical distribution of synaptic-associated proteins in the neuritic plaques of Alzheimer's disease hippocampus. Acta Neuropathol. 1994;87(2):135-42.

24. Davidsson P, Jahn R, Bergquist J, Ekman R, Blennow K. Synaptotagmin, a synaptic vesicle protein, is present in human cerebrospinal fluid: a new biochemical marker for synaptic pathology in Alzheimer disease? Mol Chem Neuropathol. 1996;27(2):195-210. doi:10.1007/BF02815094.

25. Gourmaud S, Paquet C, Dumurgier J, Pace C, Bouras C, Gray F, et al. Increased levels of cerebrospinal fluid JNK3 associated with amyloid pathology: links to cognitive decline. J Psychiatry Neurosci. 2015;40(3):151-61.

26. Mouton-Liger F, Paquet C, Dumurgier J, Lapalus P, Gray F, Laplanche IL, et al. Increased cerebrospinal fluid levels of double-stranded RNA-dependant protein kinase in Alzheimer's disease. Biol Psychiatry. 2012;71(9):829-35. doi:10.1016/j.biopsych.2011.11.031

27. Mouton-Liger F, Wallon D, Troussiere AC, Yatimi R, Dumurgier J, Magnin E, et al. Impact of cerebro-spinal fluid biomarkers of Alzheimer's disease in clinical practice: a multicentric study. J Neurol. 2014;261(1):144-51. doi:10.1007/s00415-013-7160-3.

28. McKhann GM, Knopman DS, Chertkow H, Hyman BT, Jack Jr CR, Kawas $\mathrm{CH}$, et al. The diagnosis of dementia due to Alzheimer's disease: recommendations from the National Institute on Aging-Alzheimer's Association workgroups on diagnostic guidelines for Alzheimer's disease. Alzheimers Dement. 2011;7(3):263-9. doi:10.1016/j.jalz.2011.03.005.

29. Dubois B, Feldman $\mathrm{HH}$, Jacova C, Hampel H, Molinuevo JL, Blennow K, et al. Advancing research diagnostic criteria for Alzheimer's disease: the IWG-2 criteria. Lancet Neurol. 2014;13(6):614-29. doi:10.1016/S1474-4422(14)70090-0.

30. Albert MS, DeKosky ST, Dickson D, Dubois B, Feldman HH, Fox NC, et al. The diagnosis of mild cognitive impairment due to Alzheimer's disease: recommendations from the National Institute on Aging-Alzheimer's Association workgroups on diagnostic guidelines for Alzheimer's disease. Alzheimers Dement. 2011;7(3):270-9. doi:10.1016/j.jalz.2011.03.008.

31. Sperling RA, Aisen PS, Beckett LA, Bennett DA, Craft S, Fagan AM, et al. Toward defining the preclinical stages of Alzheimer's disease: recommendations from the National Institute on Aging-Alzheimer's Association workgroups on diagnostic guidelines for Alzheimer's disease. Alzheimers Dement. 2011;7(3):280-92. doi:10.1016/j.jalz.2011.03.003.

32. Mattsson N, Andreasson U, Persson S, Arai H, Batish SD, Bernardini S, et al. The Alzheimer's Association external quality control program for cerebrospinal fluid biomarkers. Alzheimers Dement. 2011;7(4):386-95. doi:10.1016/j.jalz.2011.05.2243.e6.

33. Sudhof TC. Synaptotagmins: why so many? J Biol Chem. 2002;277(10):7629-32. doi:10.1074/jbc.R100052200

34. Ullrich B, Li C, Zhang JZ, McMahon H, Anderson RG, Geppert M, et al. Functional properties of multiple synaptotagmins in brain. Neuron. 1994;13(6):1281-91.

35. Zhang X, Rizo J, Sudhof TC. Mechanism of phospholipid binding by the C2A-domain of synaptotagmin I. Biochemistry. 1998;37(36):12395-403. doi:10.1021/big807512

36. Rufener E, Frazier AA, Wieser CM, Hinderliter A, Cafiso DS. Membrane-bound orientation and position of the synaptotagmin $\mathrm{C} 2 \mathrm{~B}$ domain determined by site-directed spin labeling. Biochemistry. 2005;44(1):18-28. doi:10.1021/ bi048370d.

37. Tapiola T, Alafuzoff I, Herukka SK, Parkkinen L, Hartikainen P, Soininen $H$, et al. Cerebrospinal fluid \{beta\}-amyloid 42 and tau proteins as biomarkers of Alzheimer-type pathologic changes in the brain. Arch Neurol. 2009;66(3): 382-9. doi:10.1001/archneurol.2008.596.

38. Buerger $\mathrm{K}$, Ewers M, Pirttila T, Zinkowski R, Alafuzoff I, Teipel SJ, et al. CSF phosphorylated tau protein correlates with neocortical neurofibrillary pathology in Alzheimer's disease. Brain. 2006;129(Pt 11):3035-41. doi:10.1093/brain/awl269.

\section{Submit your next manuscript to BioMed Central and we will help you at every step:}

- We accept pre-submission inquiries

- Our selector tool helps you to find the most relevant journal

- We provide round the clock customer support

- Convenient online submission

- Thorough peer review

- Inclusion in PubMed and all major indexing services

- Maximum visibility for your research

Submit your manuscript at www.biomedcentral.com/submit
Biomed Central 